\title{
PROPIEDADES PSICOMÉTRICAS DEL FACES IV: ESTUDIO DE VALIDEZ EN POBLACIÓN URUGUAYA
}

\section{PSYCHOMETRIC PROPERTIES OF FACES IV: VALIDITY STUDY IN URUGUAYAN POPULATION}

\author{
Daniel Costa Ball \\ Universidad Católica del Uruguay \\ Maria del Luján González Tornaría \\ Universidad Católica del Uruguay \\ Denise del Arca \\ Universidad Católica del Uruguay \\ Nuria Masjuan \\ Universidad Católica del Uruguay \\ David H. Olson \\ Universidad de Minesota, Estados Unidos
}

\begin{abstract}
Resumen: Este estudio instrumental analiza las propiedades psicométricas de una escala que evalúa el funcionamiento familiar a partir del Family Adaptability and Cohesion Evaluation Scale (FACES IV; Olson, 2011), aplicada a una muestra de 356 familias, mediante muestreo incidental en Montevideo. Se evalúa la estructura factorial, fiabilidad y validez de la versión abreviada. Se prueba mediante Análisis Factorial Confirmatorio (AFC) el modelo de 6 factores que incluye dos escalas balanceadas (cohesión y flexibilidad) y cuatro escalas desbalanceadas (caótica, rígida, desligada, y enmarañada), cada una con 4 ítems. Todas las escalas demostraron tener adecuadas propiedades psicométricas, el AFC presenta índices de ajuste aceptables $(R M S E A=.034 ; C F I=.92 ; T L I=.91 ; I F I=.92)$. Esta versión del instrumento resulta útil para la investigación del funcionamiento familiar. Es necesario realizar más estudios para mejorar su adaptación a nuestra cultura, analizando la validez de contenido en las escalas enmarañada y rígida.
\end{abstract}

Palabras Clave: funcionamiento familiar, evaluación familiar, estudio instrumental, estudio de validación, FACES IV

\begin{abstract}
This instrumental study analyses the psychometric characteristics of a scale that evaluates family functioning from the Family Adaptability and Cohesion Evaluation Scale (FACES IV; Olson, 2011), applied to a sample of 356 families, selected through incidental sampling in Montevideo. The factorial structure, reliability and validitiy of the short version, is evaluated. The model of 6 factors that includes two balanced scales (cohesion and flexibility) and four unbalanced scales (chaotic, rigid, disengaged and enmeshed), each with 4 items, is evaluated through Confirmatory Factor Analysis (CFA). All scales showed satisfactory psychometric properties, the CFA proved an adequate goodness of fit index (RMSEA $=.034 ; C F I=.92 ; T L I=.91 ; I F I=.92)$. This version of the instrument is useful for the research of family functioning. More studies are necessary to improve the adaptation to our culture, through the analysis of content validity of the enmeshed and rigid scales.
\end{abstract}

Keywords: family functioning, family assessment, instrumental study, validation study, FACES IV

\section{Introducción}

El estudio y análisis de un fenómeno tan complejo como el funcionamiento familiar requiere de instrumentos de evaluación que hayan demostrado adecuadas propiedades psicométricas en aquellos contextos en los que se usan (Carretero-Dios \& Pérez, 2007; Muñiz, Elosua y Hambleton, 2013).
Actualmente, tanto en la literatura científica como en el trabajo clínico, se reporta la complejidad de las nuevas organizaciones familiares y la diversidad de su funcionamiento (Oliva y Arranz, 2011; McGoldrick, Carter \& Garcia Preto, 2011; Rodrigo, Máiquez, Martín y Byrne, 2008; Wagner, 2011; Walsh, 2004). Por lo tanto, resulta necesario contar con instrumentos de evaluación que nos permitan investigar el 
funcionamiento familiar en nuestro medio. Para responder a esta necesidad, es fundamental identificar técnicas que hayan demostrado ser fiables y válidas y realizar el proceso de adaptación correspondiente, con el fin de controlar los sesgos de evaluación transcultural (Maglio y Schmidt, 2010). Uno de los cuestionarios de autoinforme más utilizado en la evaluación del funcionamiento familiar es el Family Adaptability and Cohesión Evaluation Scale (FACES), sustentado en el Modelo Circunflejo de los Sistemas Marital y Familiar desarrollado por Olson, Sprenkle y Russell, 1979. Dicho modelo procura explicar el funcionamiento familiar abarcando la conceptualización teórica, la investigación y la práctica clínica (Olson, 2000; Olson, Candyce \& Sprenkle, 1989). Esta escala fue modificada a lo largo de los años, mejorando sus cualidades psicométricas, conformando el FACES II (Olson, Portner, \& Bell, 1982), el FACES III (Olson, Portner, \& Lavee, 1985) y finalmente el FACES IV (Olson, 2011).

Las distintas versiones del instrumento desde el FACES hasta el FACES III, fueron aplicadas en múltiples contextos culturales, y en diversas áreas de investigación relacionadas a temas de pareja y relaciones familiares, familias con problemas especiales, sistemas familiares y salud, dinámica familiar, etc. (Kouneski, 2000). En el Río de la Plata, destaca la adaptación argentina realizada al Faces III, por Schmidt y Barreyro (2010).

En esta línea, el FACES IV, es la última versión de evaluación de auto-reporte diseñado para evaluar la cohesión y la flexibilidad familiar, que son las dos dimensiones centrales del modelo Circunflejo de los Sistemas Marital y Familiar (Olson, 2000). Este instrumento, presenta una estructura, que integra tres dimensiones que los autores encontraron relevantes en distintas teorías y también en el abordaje clínico de la familia: cohesión, flexibilidad y comunicación familiar (Olson et al., 1985). A su vez, las dos primeras dimensiones, están conformadas por seis escalas de siete ítems cada una, referidas a las dimensiones de cohesión y flexibilidad familiar: dos escalas balanceadas (cohesión balanceada y flexibilidad balanceada) y cuatro escalas desbalanceadas (enmarañada, desligada, rígida y caótica). Se contemplan de este modo los aspectos moderados y extremos de las dimensiones mencionadas. Esta última versión del instrumento, introduce el concepto de curvilinealidad en las dimensiones propuestas por el modelo, respondiendo a las críticas realizadas a las anteriores versiones. Se conforma así, una última versión que presenta una larga trayectoria a nivel internacional (Olson, 2011).

La dimensión cohesión es definida por los autores como el nivel de ligazón emocional que los miembros de la familia perciben respecto a los otros (Olson et al., 1985), focalizándose en cómo los sistemas familiares balancean la separación y la conexión (Olson \& Gorall, 2003). La dimensión flexibilidad es definida por (Olson \& Gorall, 2006) como la cualidad y expresión del liderazgo, organización, roles, reglas y negociaciones. Esta dimensión hace referencia al modo en que los sistemas balancean la estabilidad y el cambio (Olson \& Gorall, 2003). La dimensión comunicación es definida por Olson y Gorall (2006) como las habilidades de comunicación positivas utilizadas en el sistema familiar, siendo ésta la dimensión facilitadora del sistema. Satisfacción familiar refiere a la realización y la felicidad con el funcionamiento general de la familia, específicamente en cuanto al nivel de cohesión y flexibilidad, percibidos por cada integrante (Olson \& Gorall, 2006).

Las escalas del FACES IV resultaron fiables y válidas, presentando altos niveles de validez concurrente, de constructo y discriminante para población de Estados Unidos de América (Olson, 2000; Olson, 2011). En los últimos años, han comenzado a proliferar estudios internacionales con el FACES IV: análisis de fiabilidad y validez para Italia (Loriedo, Di Nuovo, \& Visani, 2013); y de validez factorial y de constructo en adolescentes italianos (Baiocco, Cacioppo, Laghi \& Tafa, 2012), estudios de aplicabilidad transcultural en Hungría (Mirnics, Vargha, Tóth, \& Bagdy, 2010); adaptación transcultural del FACES IV a Grecia (Koutra, Trilivia, Rouneliataki, Lionis \& Vgontzas, 2012) y en el ámbito español, adaptación del FACES IV a España (Rivero, Martínez-Pampliega, \& Olson, 2010). De los estudios presentados anteriormente, solamente los análisis de validez de constructo realizados por Olson (2011) a través de un Análisis Factorial Confirmatorio (AFC) ratifica la estructura conceptual del Modelo Circunflejo de los Sistemas Marital y Familiar, como una estructura de seis dimensiones. Únicamente el estudio de Rivero et al. (2011) replica los resultados de una estructura factorial de seis factores como la hallada por Olson (2011), pero usando una escala abreviada. El resto de los estudios, de validez de constructo del FACES IV han 
arribado a conclusiones similares pero usando un Análisis Factorial Exploratorio (AFE) y no un AFC. En los distintos países donde se aplicó, el modelo no ajusta al original propuesto por Olson (2011), tanto en Italia, Hungría y España (Loriedo et al., 2013; Mirnics et al., 2010; Rivero et al., 2010; Sanz, Iraurgi, Martínez-Pampliega, 2002). En los dos primeros países nombrados, a pesar que el modelo original no ajustó en el proceso de adaptación, se continuó trabajando con la escala total sin arribar a una escala o versión reducida. En el caso italiano, para mejorar el ajuste inicial, se retomó el modelo original de Olson (2011) incluyendo las escalas de Comunicación y Satisfacción. Se obtuvo así, una mejora del ajuste inicial de los datos al modelo, pero no se logró replicar los resultados reportados por Olson (2011). En las conclusiones se reconoce la existencia de dos factores correspondientes a las escalas balanceadas, cuatro factores para las cuatro escalas desbalanceadas y dos escalas unidimensionales: comunicación y satisfacción familiar (Loriedo et al., 2013). En el segundo caso, en Hungría, no se utilizó un AFC, sino un AFE, y se evidenció una estructura de cinco factores. En el primer factor confluyen ítems de cohesión, flexibilidad junto a cinco de desligada con carga negativa. Un segundo factor compuesto por los restantes ítems de flexibilidad; y los restantes tres relacionados con el factor enmarañado, rígido y caótico. De los análisis con las escalas de las dimensiones cohesión y flexibilidad, concluyen que existe evidencia de dos escalas balanceadas y cuatro desbalanceadas, coincidiendo con lo reportado por Olson (2011). En el proceso de adaptación del FACES IV al español (Rivero et al., 2010), una vez de comprobado que el modelo no ajustaba, se realizaron rigurosos estudios psicométricos y se arribó a una escala abreviada. Se administró a una muestra de 455 estudiantes universitarios, y se llegó a un modelo de 6 escalas como el propuesto por los autores, pero compuesto por una versión reducida de cuatro ítems por escala.

En Uruguay, en el 2009 se realizó el estudio piloto en el proceso de adaptación del FACES IV, encontrando que los índices de consistencia interna de las escalas pertenecientes a las dimensiones cohesión y flexibilidad familiar, resultaron moderados a buenos, pero ubicándose por debajo de los reportados originalmente por los autores de la escala (Costa Ball et al., 2009). Los estudios de unidimensionalidad de cada una de las escalas del FACES IV, evidenciaron problemas únicamente en la escala enmaraña$\mathrm{da}$, presentando un mal ajuste en un AFC y una fiabilidad baja, usando los siete ítems originales de la escala $(\alpha=.67)$. El resto de las escalas ajustaron a un modelo de factor único, evidenciando fiabilidades entre .70 y .78 (Costa Ball, et al., Marzo, 2010). Desde esa fecha, nuestro equipo ha seguido aplicando el FACES IV en el marco de investigaciones realizadas en los trabajos finales de Grado, Postgrado y Maestría en nuestra Facultad de Psicología (Brown, 2011; del Arca, 2010; Feibuscheurez, 2009; Lestido, 2013; Massonnier, 2011; Urrutia, 2012).

Continuando con esta línea de análisis sobre las propiedades psicométricas del FACES IV, este estudio instrumental (Montero y León, 2002) pretende examinar la dimensionalidad, fiabilidad y validez de la versión uruguaya. Para ello, y siguiendo las recomendaciones de Carretero-Dios y Pérez (2007) y Muñiz, Elosua y Hambleton (2013), se pone a prueba en primer lugar el modelo factorial de seis factores del FACES IV (Olson, 2011). En segundo lugar, al no ajustar, se pone a prueba la versión abreviada del FACES IV de España (Rivero et al., 2010). En tercer lugar, al no alcanzar el ajuste, se comienza con el proceso de adaptación del FACES IV al Uruguay con el objetivo de arribar a una escala abreviada que presente adecuadas propiedades psicométricas, es decir, se evalúan su validez (de constructo, concurrente) $y$ fiabilidad.

\section{Método}

\section{Participantes}

Se seleccionó en primer lugar una muestra de 356 familias distribuidas en instituciones de educación privada de Montevideo, alcanzando un total de 970 sujetos. A los efectos de este estudio, se seleccionó al azar a un integrante de cada familia, conformando una muestra de 356 sujetos. El análisis de datos que se presenta en este documento refiere a la percepción que esos sujetos tienen sobre sus relaciones familiares.

Las edades de los participantes se encuentran comprendidas entre 12 y 95 años $(M=34.28$; $D T=15.35)$. El $36 \%$ de los sujetos pertenecen a instituciones emplazadas en zonas con un nivel socioeconómico medio bajo, $24 \%$ con un nivel medio y $40 \%$ con un nivel medio alto. 
El $57.6 \%$ de la muestra es de sexo femenino y el $42.4 \%$ de sexo masculino. En cuanto a su rol, el $43 \%$ son madres $(M=40.54$; $D T=6.96)$, el $33 \%$ padres $(M=42.57$; $D T=7.31)$, el $19.5 \%$ hijos $(M=14.07$; $D T=3.39)$ y $4.5 \%$ otros familiares que viven bajo el mismo techo $(M=55.46 ; D T=22.46)$.

\section{Procedimiento}

Se trabajó mediante un muestreo por conglomerados bietápico. En la primera etapa se escogieron 8 instituciones de educación privada de Montevideo. Se utilizó el procedimiento incidental para obtener una muestra representativa de los diferentes niveles socioeconómicos de la ciudad (bajo, medio y alto). Se administraron los protocolos a los 970 sujetos que aceptaron participar. En todos los casos se trabajó con consentimientos, en primer lugar institucional y luego de las familias, para participar de la investigación.

Con el fin de realizar el AFC y no contradecir el supuesto de independencia, se seleccionó al azar a un participante de cada familia, alcanzando una muestra total de 356 sujetos. Siguiendo a Hair (como se citó en Lévy, Martín y Román, 2006), se recomienda que al menos se cuente con 5 encuestados por parámetro, lo que quedaría cubierto con los 356 participantes.

\section{Instrumentos}

- Family Adaptability and Cohesion Evaluation Scale (FACES IV; Olson 2011). Se compone de 6 escalas de 7 ítems cada una, alcanzando un total de 42 ítems. Las 2 escalas balanceadas (cohesión balanceada y flexibilidad balanceada) corresponden a los aspectos moderados/ saludables de las dimensiones cohesión y flexibilidad. Las 4 escalas desbalanceadas corresponden a los aspectos extremos en las dimensiones de cohesión familiar (desligada y enmarañada) y de flexibilidad familiar (rígida y caótica). Se contesta con una escala likert de 5 puntos por todos los miembros de la familia mayores de 12 años.

- Satisfacción Familiar (FSS, Olson, 1995). Esta escala presenta 10 ítems y evalúa la satisfacción de los miembros de la familia respecto a la cohesión, flexibilidad y comunicación familiar, las 3 dimensiones del Modelo Circunflejo.

\section{Análisis de datos}

Para realizar los estudios psicométricos, se tomó como referencia los análisis propuestos por Olson (2011) y Rivero et al. (2010). Se analizó la validez de constructo con la finalidad de descartar una estructura unidimensional con los 42 ítems del FACES IV, y poder dar apoyo a la estructura propuesta por los autores, de un modelo de seis factores.

Análisis de ítems, se realizó para comprobar si los ítems ajustaban adecuadamente en la escala correspondiente. Se utilizaron los siguientes criterios para la eliminación: a) que el promedio del ítem sea de una desviación estándar más o menos de la media de la escala, b) que los ítems tengan una desviación estándar reducida (<.05); c) que el coeficiente de correlación de Pearson entre el ítem y la escala al que pertenece sea menor a .40, d) que el coeficiente alfa de Cronbach aumente en más de .03 puntos si los ítems son eliminados, e) que las escalas no tengan un número diferente de ítems, con el fin de homogeneizarlas.

Análisis Factorial Confirmatorio, se realizó el AFC con AMOS 18 para evaluar el ajuste de los datos a los distintos modelos estudiados. Para la evaluación del modelo global, utilizamos tres tipos de medidas de ajuste global; medidas de ajuste absoluto, parsimonioso e incremental. Las medidas de ajuste absoluto evalúan el grado de exactitud en que el modelo global predice correctamente la matriz de covarianza observada: Índice de la Chi-cuadrado (Chi squared, $\chi^{2}$ ) y el error de aproximación cuadrático medio (Root Mean Square Error of Aproximation; RMSEA). Las medidas de ajuste incremental utilizadas fueron: índice de ajuste normalizado (Normed Fit Index, $N F I$ ), índice de ajuste no normalizado (Tucker Lewis Index, TLI) e índice de ajuste comparativo (Comparative Fit Index, CFI). Finalmente como índices de evaluación de parsimonia se utilizaron el índice de Chi-cuadrado normalizada (Normed Chi-squared, NCS) o el ratio Chicuadrado por grados de libertad $\left(\chi^{2} / g l\right)$. Para el índice Chi-cuadrado un 1- $\alpha=0.95$, una probabilidad de $\chi^{2}>.05$ indica un buen ajuste. Se señala que este índice es muy sensible al tamaño muestral: cuando la muestra es mayor a 200 sujetos, el test puede no ser fiable (Bentler \& Bonnet, 1980). El nivel adecuado para un buen ajuste del modelo es un RMSEA inferior a .08 (MacCallum, Browne \& Sugawara, 1996), 
alcanzando un muy buen ajuste para valores inferiores a .06 (Hu \& Bentler, 1999) y un límite superior estricto de .07 (Steiger, 2007). Para $\mathrm{NFI}, \mathrm{TLI}$ y $\mathrm{CFI}$, valores mayores o iguales a .90 serían indicativos de un ajuste adecuado (Bentler \& Bonnet, 1980). Por último, para el ratio $\left(\chi^{2} / g l\right)$, valores inferiores a 2 indican un buen ajuste (Lévy et al., 2006).

Reespecificación de un modelo de AFC. En primer lugar, se da el proceso de especificación que tiene como objetivo establecer formalmente el modelo. Luego, se pasa a la identificación, estimación de parámetros, ajuste del modelo, interpretación y reespecificación. El objetivo es mejorar la bondad de ajuste del modelo evaluado. Para ello se tiene en cuenta el Test de significación de parámetros, según el cual los parámetros que no sean estadísticamente significativos son candidatos a ser eliminados o fijados. Los Índices de modificación, informan sobre el cambio esperado en el valor chi-cuadrado si se libera un determinado parámetro fijo y se estima de nuevo. Valores de índices de modificación mayores a 3.84 indican que se produce una disminución estadísticamente significativa en el valor chi-cuadrado (Lévy et al., 2006).

Análisis de dimensionalidad de la escala satisfacción familiar, se utilizó el AFE con SPSS 18.0 para Windows, utilizando la técnica de extracción de ejes principales. Para confirmar el número de factores a extraer se utilizó la regla de Kaiser-Guttman, la interpretación del gráfico Scree Test (Cattell, 1966) y el Análisis Paralelo (Horn, 1965). Para analizar la consistencia interna de la escala, se obtuvieron los coeficientes alfa de Cronbach para la escala.

Para el análisis de la validez concurrente, se realizó una correlación entre las escalas de satisfacción y las escalas del FACES IV. Todos los análisis se realizaron con SPSS 18, excepto los AFC, para los cuales se utilizó el paquete estadístico AMOS 18.

\section{Resultados}

Se corrió el AFC con la finalidad de verificar la adecuación de la estructura original de seis factores y replicar los resultados reportados por Olson (2011). Se utilizaron todos los ítems originales ( 7 ítems por escala) que conforman el FACES IV. Los resultados obtenidos muestran que con los datos uruguayos el modelo no ajusta a una estructura de seis factores, como la propuesta por los autores: $\chi^{2}(804$, $N=294)=1509,164 ; p=.000 ; \chi^{2} / g l=1.87$; $R M S E A=.055 ; \mathrm{NFI}=.53 ; \mathrm{IFI}=.70 ; \mathrm{CFI}=.70$; $T L I=.67$. En segundo lugar, replicamos la versión abreviada al español de Rivero et al. (2010), con los datos obtenidos con la muestra uruguaya. Los resultados evidencian que el modelo tampoco ajusta a una estructura de seis factores utilizando cuatro ítems por escala: $\chi^{2}(237, N=294)=384,640 ; p=.000$; $\chi^{2} / \mathrm{gl}=1.62 ; \mathrm{RMSEA}=.046 ; \mathrm{NFI}=.72$; $I F I=.87 ; C F I=.87 ; T L I=.84$.

A la luz de estos resultados y siguiendo la estrategia de análisis planteada por Rivero et al. (2010), se recomienda continuar con los análisis de la estructura interna del cuestionario, agrupando los ítems según a qué escala pertenece originalmente en el Modelo.

Escalas Balanceadas centrales (cohesión / flexibilidad). Teniendo en cuenta el Modelo Circunflejo, se toman todos los ítems que conforman las escalas cohesión y flexibilidad balanceadas y se estudia su dimensionalidad. Se pone a prueba la hipótesis de factor único y de dos factores. Los resultados no ajustan a un modelo de factor único (ver Tabla 1/I). En segundo lugar, se configura un modelo de dos dimensiones y se lo pone a prueba. Los resultados evidencian un mal ajuste al modelo con una estructura de dos dimensiones (ver Tabla 1/II). A continuación, se analiza cada escala por separado para comprobar la unidimensionalidad, eliminar ítems

Tabla 1

Índices de bondad de ajuste del modelo inicial y reespecificado (modelo bifactorial) de las escalas centrales balanceadas (cohesión / flexibilidad)

\begin{tabular}{ccccccccc}
\hline \multirow{2}{*}{ Pasos } & Modelo & gl & $\chi^{2}$ & $\chi^{2} / g l$ & RMSEA & CFI & TLI & IFI \\
\hline I & Factor único & 77 & $158.722^{* * *}$ & 2.061 & .060 & .869 & .845 & .872 \\
II & Dos factores & 76 & $153.232^{* * *}$ & 2.016 & .059 & .876 & .856 & .879 \\
\hline
\end{tabular}

Nota. ${ }^{*} p<.05 ;{ }^{* *} p<.01 ;{ }^{* * *} p<.001$ 
que atenten contra la misma y a su vez, poder reespecificar el modelo de dos factores para mejorar el ajuste.

Escala balanceada de Cohesión. Se corre un AFC para comprobar la unidimensionalidad de la escala, los resultados muestran un ajuste pobre (ver Tabla 2/I). Para reespecificar el modelo y mejorar el ajuste, se elimina el ítem 19, mejorando la fiabilidad total de la escala en más de .30 puntos. Los ítems 25 y 37 se eliminan por presentar valores de correlación entre el ítem y la escala menores a .40. Tras la eliminación de los ítems, se corre un AFC para verificar la unidimensionalidad de la escala. Los resultados evidencian un muy buen ajuste al modelo de factor único (ver Tabla 2/ll y Tabla 3).

Escala de Flexibilidad Balanceada. Los resultados del AFC evidencian un ajuste adecuado. Eliminando ítems, se puede mejorar el ajuste al reespecificar el modelo (ver Tabla 2/III). Se elimina el ítem 8 por presentar una comunalidad baja y ser la variable menos explicada por el modelo. Se elimina el ítem 38 por tener un coeficiente de asimetría alto en relación a los otros ítems (As = -1.84). Por último, se elimina el ítem 32 para homogenizar la cantidad de ítems por escala, al presentar la carga factorial más baja $(<.40)$. Con los cuatro ítems restantes $(2$, 14,20 y 26) se corre nuevamente el AFC y los resultados evidencian un muy buen ajuste al modelo de factor único (ver Tabla 2/IV y Tabla 3).

Tras eliminar los ítems que atentaban contra la unidimensionalidad (ítems 8, 19, 25, 32, 37,
38) de cada una de las escalas balanceadas (cohesión y flexibilidad) y reespecificar el modelo, volvemos a someterlo a prueba a través de un AFC obligando obtener dos factores correlacionados. Los resultados evidencian un buen ajuste al modelo de dos factores: $\chi^{2}(237, \mathrm{~N}=294)=22,909 ; \mathrm{p}=.000 ; \chi^{2} / \mathrm{gl}=1.20$; $\mathrm{RMSEA}=.026 ; \mathrm{IFI}=.98 ; \mathrm{CFI}=.98 ; \mathrm{TLI}=.98$.

Escalas Desbalanceadas (desligada, enmarañada, rígida y caótica). Se toman las cuatro escalas desbalanceadas del Modelo Circunflejo. De la dimensión cohesión, los extremos desbalanceados desligado y enmarañado; de la dimensión flexibilidad, los extremos desbalanceados rígido y caótico. Con estas cuatro escalas, se corre un AFC para comprobar si el modelo ajusta a la solución de cuatro factores propuesta por Olson (2011). Los resultados muestran que los datos no ajustan: $\chi^{2}$ (344, $\mathrm{N}=294)=674.972 ; \mathrm{p}=0.000 ; \chi^{2} / \mathrm{gl}=1.96$; RMSEA $=.057 ; \mathrm{IFI}=.74 ; \mathrm{CFI}=.73 ; \mathrm{TLI}=.70$. Con el objetivo de mejorar el ajuste, se intenta clarificar la estructura de cuatro factores y, para homogeneizar cada escala a un número igual de ítems, se decide seguir la estrategia de análisis sugerida en Rivero et al. (2010) y trabajar con los ítems agrupados según la dimensión a la que pertenecen.

\section{Dimensión cohesión desbalanceada.} Con los ítems de las escalas desligada y enmarañada, se corrió un AFC exigiendo que extraiga una solución de dos factores. Los resultados no ajustan a una solución

Tabla 2

Índices de bondad de ajuste del modelo inicial y reespecificado de las escalas balanceadas (cohesión / flexibilidad)

\begin{tabular}{|c|c|c|c|c|c|c|c|c|c|}
\hline Pasos & $\begin{array}{c}\text { Ítems } \\
\text { eliminados }\end{array}$ & Modelo & $g l$ & $\chi^{2}$ & $\chi^{2} / g l$ & RMSEA & CFI & $T L I$ & $I F I$ \\
\hline \multicolumn{10}{|c|}{ Escala cohesión balanceada } \\
\hline I & & Factor único & 14 & $35.6^{* *}$ & 2.54 & .073 & .931 & .89 & .932 \\
\hline II & $19,25,37$ & Reespecificado & 2 & .58 & 2.92 & .000 & 1.00 & 1.10 & .975 \\
\hline \multicolumn{10}{|c|}{ Escala flexibilidad balanceada } \\
\hline III & & Factor único & 14 & $21.4^{* * *}$ & 1.53 & .043 & .947 & .921 & .950 \\
\hline IV & $8,32,38$ & Reespecificado & 2 & .58 & 2.92 & .000 & 1.00 & 1.10 & 1.03 \\
\hline
\end{tabular}

Tabla 3

Índices de bondad de ajuste del modelo inicial y reespecificado de la escala desligada

\begin{tabular}{|c|c|c|c|c|c|c|c|c|c|}
\hline Pasos & $\begin{array}{c}\text { Ítems } \\
\text { eliminados }\end{array}$ & Modelo & $g l$ & $\chi^{2}$ & $\chi^{2} / g l$ & RMSEA & CFI & $T L I$ & IFI \\
\hline I & & Factor único & 14 & $28.504^{*}$ & 2.036 & .059 & .994 & .917 & .946 \\
\hline II & $39,15,21$ & Reespecificado & 9 & 0.060 & 0.030 & .000 & 1.00 & 1.073 & 1.02 \\
\hline
\end{tabular}


bifactorial como la propuesta por los autores: $\chi^{2}(8, N=294)=674.972 ; p=0.000 ;$ $\chi^{2} / \mathrm{gl}=1.96 ; \mathrm{RMSEA}=.057 ; \mathrm{CFI}=.73$; $\mathrm{TLI}=.70$. En consecuencia, se estudia la unidimensionalidad de cada una de las dos escalas (desligada / enmarañada) usando un AFC.

Desligada. Se corrió el AFC, con los siete ítems que conforman la escala, logrando un buen ajuste a un modelo unidimensional (ver Tabla 3/l). Se elimina el ítem 39 para mejorar la fiabilidad de la escala en más de 3 puntos. Se corre un nuevo análisis y se obtiene una solución unidimensional mejorando el ajuste del modelo anterior. Las cargas de los seis ítems restantes, son superiores al criterio de selección de ítems (> .40). Para cumplir el criterio de homogeneizar la cantidad de ítems por escala, se elimina el ítem 21 que presenta un alto índice de modificación y en el AFC subsiguiente se elimina el ítem 15 por la misma razón. En suma, la escala desligada perdió tres de los siete ítems originales, arribando a una escala unidimensional de cuatro ítems, con un excelente ajuste (ver Tabla 3/II), quedando conformada por los ítems: 3, 9, 27, 33 y una fiabilidad de .56 .

Enmarañada. Se corre el AFC, con los siete ítems que conforman la escala, los resultados obtenidos no ajustan a un modelo de estructura de factor único (ver Tabla 4/I). Para mejorar el ajuste, se debe modificar el modelo a causa de errores de especificación interna. Los índices de modificación arrojados en el AFC, permiten detectar la disminución mínima en el valor $\chi^{2}$ de ajuste del modelo, si un parámetro fijo se hiciera libre y se volviera a estimar el modelo.
Se consideran altos los índices de modificación superiores a 3.84. Puesto que los ítems 10 , 34 y 40 presentan índices superiores al valor de referencia, se eliminan. Reespecificado el modelo, se corre nuevamente el análisis, obteniéndose un muy buen ajuste (ver Tabla 4/ II). En suma, la escala enmarañada perdió tres de los siete ítems originales, arribando a una escala unidimensional de cuatro ítems, con un excelente ajuste, quedando conformada por los ítems 4, 16, 22, 28 y con una fiabilidad de .60.

\section{Dimensión flexibilidad desbalanceada.} Con los ítems de las escalas rígida y caótica, se corrió un AFC exigiendo que extraiga tanto una solución monofactorial como de dos factores. Los resultados no ajustan a un modelo unidimensional (ver Tabla 5/I) ni a un modelo bifactorial (ver Tabla 5/II). En consecuencia, se estudia la unidimensionalidad de cada una de las escalas (rígida / caótica), por separado.

Rígida. Se corre el AFC y los resultados no ajustan a un modelo unidimensional (ver Tabla 6/I). Se elimina el ítem 29 para mejorar la fiabilidad de la escala en más de .3 puntos. Se corre un nuevo análisis y se obtiene una solución unidimensional mejorando apenas el ajuste del modelo anterior (ver Tabla 6/II). Se elimina el ítem 23 por presentar la menor carga factorial de los cinco restantes, logrando así un muy buen ajuste a un modelo unidimensional (ver Tabla 6/ III). Para cumplir el criterio de homogeneizar la cantidad de ítems por escala, se elimina el ítem 35 que presenta la carga factorial más baja de los cuatro restantes. En suma, la escala rígida perdió tres de los siete ítems originales, arribando

Tabla 4

Índices de bondad de ajuste del modelo inicial y reespecificado de la escala enmarañada

\begin{tabular}{ccccccccccc}
\hline \multirow{2}{*}{ Pasos } & $\begin{array}{c}\text { Ítems } \\
\text { eliminados }\end{array}$ & Modelo & gl & $\chi^{2}$ & $\chi^{2} / g l$ & RMSEA & NFI & CFI & TLI & IFI \\
\hline I & Factor único & 14 & $47.73^{* * *}$ & 3.41 & .091 & .74 & .794 & .690 & .802 \\
II & $10,34,40$ & Reespecificado & 2 & .067 & 0.33 & .000 & .99 & 1.00 & 1.03 & 1.01 \\
\hline Nota. ${ }^{*} \mathrm{p}<.05 ;{ }^{* *} \mathrm{p}<.01 ;{ }^{* * *} \mathrm{p}<.001$ & & & & & & & & &
\end{tabular}

Tabla 5

Índices de bondad de ajuste del modelo inicial y reespecificado de las escalas desbalanceadas de la dimensión flexibilidad

\begin{tabular}{ccccccccccc}
\hline \multirow{2}{*}{ Pasos } & $\begin{array}{c}\text { Ítems } \\
\text { eliminados }\end{array}$ & Modelo & gl & $\chi^{2}$ & $\chi^{2} / g l$ & RMSEA & NFI & CFI & TLI & IFI \\
\hline I & & Factor único & 17 & $277.11^{* * *}$ & 3.59 & .094 & .54 & .61 & .54 & .62 \\
II & & Dos factores & 76 & $191.00^{* * *}$ & 2.51 & .072 & .68 & .77 & .73 & .78 \\
II & \multirow{2}{*}{$10,34,40$} & $\begin{array}{c}\text { Dos factores } \\
\text { Reespecificado }\end{array}$ & 2 & & .67 & .33 & .000 & .99 & 1.00 & 1.0 \\
\hline
\end{tabular}

Nota. ${ }^{*} p<.05 ;{ }^{* *} p<.01 ;{ }^{* * *} p<.001$ 
a una escala unidimensional de cuatro ítems, con un excelente ajuste (ver Tabla 6/IV), quedando conformada por los ítems 5, 11, 17, 41 y una fiabilidad de .57 .

Caótica. Se corre el AFC logrando un buen ajuste a un modelo unidimensional (ver Tabla $7 / I$ ). Eliminamos el ítem 30 para mejorar la fiabilidad de la escala en más de .3 puntos (ver Tabla $7 /$ II). Se corre un nuevo análisis y se obtiene una solución unidimensional mejorando el ajuste del modelo anterior. Se elimina el ítem 36 por ser uno de los que presenta la carga más baja de los cinco restantes, el ajuste empeora apenas (ver Tabla 7/III). Por último, se elimina el ítem 24 para cumplir con el criterio de homogeneizar el número de ítems por escala, eliminando el que carga menos de los restantes. En suma, la escala caótica perdió tres de los siete ítems originales, arribando a una escala unidimensional de cuatro ítems, con un excelente ajuste (ver Tabla 7/IV), quedando conformada por los ítems $6,12,18$, 42 y una fiabilidad de .65 .

Tras eliminar los ítems que atentaban contra la estructura de cuatro factores en las escalas desbalanceadas, se realiza la reespecificación del modelo y volvemos a someterlo a prueba como un modelo de cuatro factores correlacionados. Los resultados del AFC evidencian un buen ajuste al modelo de cuatro factores: $\chi^{2}(98, N=294)=136.77$; $\mathrm{p}=0.006 ; \chi^{2} / \mathrm{gl}=1.39 ; \mathrm{RMSEA}=.037 ; \mathrm{NFI}=.79$; $\mathrm{IFI}=.93 ; \mathrm{CFI}=.930 ; \mathrm{TLI}=.913$.
Análisis Factorial Confirmatorio con el modelo total reespecificado. Como resultado de los análisis anteriores, se reespecifica el modelo de seis factores propuesto por los autores, pasando de los 42 ítems originales a 24 ítems en la versión uruguaya. La Figura 1 muestra los resultados del AFC poniendo a prueba la hipótesis de la estructura de seis factores, usando la escala abreviada que representa las escalas que pertenecen a las dimensiones de cohesión y flexibilidad del FACES IV. Los factores ubicados en los ángulos superior e inferior de la figura, representan los extremos desbalanceados en ambas dimensiones, enmarañada y desligada en la dimensión cohesión, caótica y rígida, en la dimensión flexibilidad. Las escalas balanceadas para las dimensiones cohesión y la flexibilidad se presentan en el centro de la figura. Los estadísticos de ajuste sugieren un ajuste aceptable. $\chi^{2}(237, \mathrm{~N}=294)=318.45 ; \mathrm{p}=0.000$; $\chi^{2} / \mathrm{gl}=1.34 ; \mathrm{RMSEA}=.034 ; \mathrm{IFI}=.92$; $\mathrm{CFI}=.92 ; \mathrm{TLI}=.91$.

Esta figura también muestra las cargas factoriales para los 24 ítems en sus respectivas escalas. Las cargas factoriales en la escala de cohesión balanceada promediaron .62 (rango .59 a .67). Las cargas factoriales en el extremo alto de cohesión (enmarañada) promediaron .51 (rango .23 a .73) y en el extremo bajo de la cohesión (desligada) promediaron .49 (rango .46 a .53). Las cargas factoriales en la escala flexibilidad balanceada promediaron .42 (rango

Tabla 6

Índices de bondad de ajuste del modelo inicial y reespecificado de la escala rígida

\begin{tabular}{|c|c|c|c|c|c|c|c|c|c|c|}
\hline Pasos & $\begin{array}{c}\text { Ítems } \\
\text { eliminados }\end{array}$ & Modelo & $g l$ & $\chi^{2}$ & $\chi^{2} / g l$ & RMSEA & $I F I$ & $C F I$ & $T L I$ & $I F I$ \\
\hline I & & Factor único & 14 & $48.88^{* * *}$ & 3.13 & .085 & .781 & .839 & .758 & .845 \\
\hline II & 29 & Reespecificado & 9 & $19.91^{*}$ & 2.216 & .064 & .934 & .932 & .887 & .935 \\
\hline III & 29,23 & Reespecificado & 5 & 6.73 & 1.34 & .034 & .987 & .987 & .974 & .987 \\
\hline IV & $29,23,35$ & Reespecificado & 2 & 0.93 & 0.46 & .000 & 1.01 & 1.00 & 1.038 & 1.01 \\
\hline
\end{tabular}

Nota. ${ }^{*} p<.05 ;{ }^{* *} p<.01 ;{ }^{* * *} p<.001$

Tabla 7

Índices de bondad de ajuste del modelo inicial y reespecificado de la escala caótica

\begin{tabular}{ccccccccccc}
\hline Pasos & $\begin{array}{c}\text { Ítems } \\
\text { eliminados }\end{array}$ & Modelo & gl & $\chi^{2}$ & $\chi^{2} / g l$ & RMSEA & IFI & CFI & TLI & IFI \\
\hline I & & Factor único & 14 & 21.145 & 1.51 & .042 & .972 & .971 & .956 & .972 \\
II & 30 & Reespecificado & 9 & 13.06 & 1.45 & .039 & .983 & .983 & .972 & .983 \\
III & 30,36 & Respecificado & 5 & 10.471 & 2.094 & .061 & .972 & .971 & .942 & .972 \\
IV & $30,36,24$ & Respecificado & 2 & 0.074 & 0.037 & .000 & 1.01 & 1.00 & 1.042 & 1.01 \\
\hline \multicolumn{2}{r}{ Nota. ${ }^{*} p<.05 ;{ }^{* *} p<.01 ;{ }^{* * *} p<.001$}
\end{tabular}


.32 a .58). Por otra parte, las cargas factoriales en el extremo alto de flexibilidad (caótica) promediaron .56 (rango .45 a .70) y en el extremo bajo de flexibilidad (rígida) promediaron .50 para la escala desligada (rango .44 a .61).

Las flechas curvas de doble punta representan las correlaciones entre cada escala del modelo. La escala cohesión balanceada presenta una fuerte correlación negativa con la escala desligada $(r=-.93)$ y correlaciona negativamente pero poco con la escala enmarañada $(r=-.15)$. La escala flexibilidad balanceada presenta una fuerte a moderada correlación negativa con la escala desligada $(r=-.57)$ y correlaciona positivamente pero poco con la escala enmarañada $(r=.17)$. Las escalas extremas desbalanceadas no están correlacionadas entre sí. Dos escalas de la dimensión cohesión no correlacionan (desligada y enmarañada, $r=.02$; varianza compartida $=.007$ ), lo mismo ocurre para dos escalas de la dimensión flexibilidad (rígida y caótica $r=.02$; varianza compartida $=009$ ). Estos resultados son la prueba de la independencia de las escalas desbalanceadas (enmarañada, desligada, rígida y caótica) para los extremos desbalanceados de cohesión y flexibilidad, resultando esto congruente con los resultados obtenidos por Olson (2011). En la Tabla 8 se muestran los estadísticos descriptivos de las escalas.

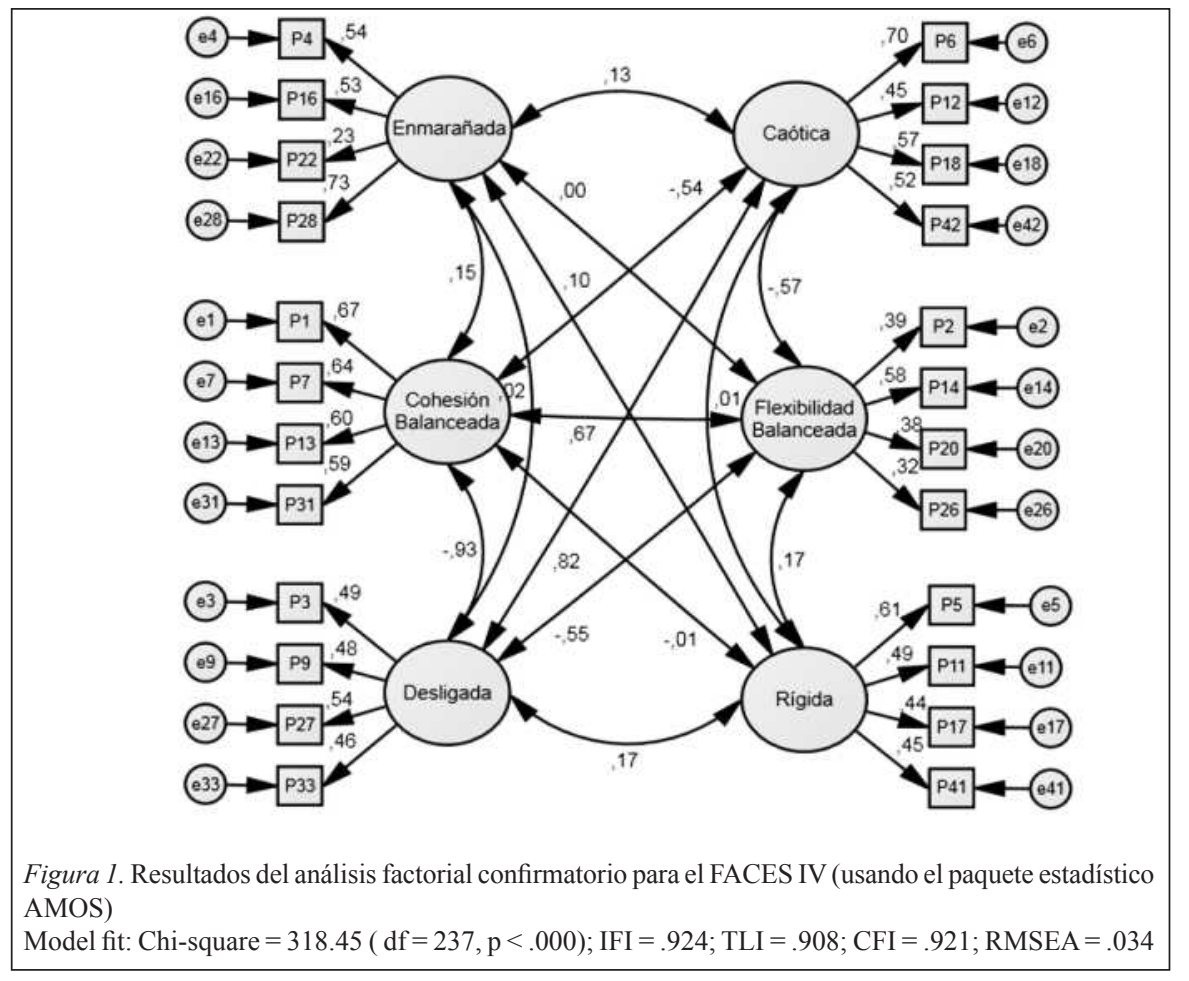

Tabla 8

Promedio en cada una de las escalas del FACES IV y desvio típico

\begin{tabular}{lcc}
\hline & \multicolumn{2}{c}{ Población normativa } \\
\cline { 2 - 3 } Escalas del FACES IV & $M$ & $D E$ \\
\hline Cohesión Balanceada & 18,19 & 2,11 \\
Flexibilidad Balanceada & 15,84 & 2,49 \\
Desligada & 6,32 & 2,66 \\
Enmarañada & 10,62 & 3,35 \\
Rígida & 11,31 & 3,40 \\
Caótica & 7,58 & 3,00 \\
Comunicación & 41,28 & 5,95 \\
Satisfacción & 41,50 & 5,67 \\
\hline
\end{tabular}


Análisis factorial de la escala de Satisfacción familiar. Los resultados del AFE, sobre la bondad de ajuste de los datos a un modelo factorial, revelaron que la medida de adecuación muestral de KaiserMeyer-Olkin $(\mathrm{KMO}=0.89)$ y la prueba de esfericidad de Bartlett $\left(\chi^{2}=1078.175\right.$; $\mathrm{df}=45$; $p=0.000$ ) evidenciando la posibilidad de realizar el AFE, el cual se efectuó a través del método de extracción de Componentes Principales. Se utilizó la regla de Kaiser-Guttman, la interpretación del gráfico Scree Test (Cattell, 1966) y el Análisis Paralelo (Horn, 1965), todos indicaron la conveniencia de interpretar únicamente un factor (ver Tabla 9).

Se extrajo un único factor que explicó el $47 \%$ de la varianza. Como criterios complementarios se decidió retener aquellos ítems con una saturación factorial superior a .4. Como resultado se obtuvo una solución de 10 ítems distribuidos en un único factor. Esto fue confirmado tanto por el gráfico Scree como por el Análisis Paralelo.
Análisis de correlación de las escalas. Se llevó a cabo un análisis de correlación para evaluar las relaciones entre las seis escalas del FACES IV (ver Tabla 10). Las dos escalas centrales de cohesión y flexibilidad alcanzaron una correlacionaron positiva y fuerte $(r=.67)$.

Fiabilidad de las seis escalas. Se evaluó la consistencia interna de las seis escalas del FACES IV, la fiabilidad resultante es la siguiente: cohesión balanceada $=.71$, enmarañada $=.60$, desligada $=.56$, flexibilidad balanceada $=.46$, caótica $=.65$, rígida $=.57$.

\section{Discusión}

El objetivo de este estudio fue obtener una adaptación de la escala FACES IV al Uruguay, replicando los análisis de validez de constructo presentados por Rivero et al. (2010) y Olson (2011). Se utilizaron rigurosos procedimientos de análisis estadístico y psicométrico en todas las

Cargas factoriales del análisis de componentes principales: comunalidades, valores eigen y porcentaje de la varianza

\begin{tabular}{lcc}
\hline \multicolumn{1}{c}{ Reactivo } & & \\
\hline \multicolumn{1}{c}{ Carga factorial } & Comunalidades \\
\hline 53. El grado de cercanía entre los miembros de la familia & .69 & .47 \\
54. La habilidad de su familia para enfrentar y resolver los problemas & .71 & .50 \\
55. La habilidad de su familia para ser flexible & .68 & .47 \\
56. La habilidad de su familia para compartir experiencias positivas & .67 & .45 \\
57. La calidad de la comunicación entre los miembros de su familia & .72 & .52 \\
58. La habilidad de su familia para resolver conflictos & .74 & .55 \\
59. La cantidad de tiempo que pasan juntos como familia & .47 & .23 \\
60. La manera en que los problemas son discutidos & .77 & .59 \\
61. La justicia de las criticas en su familia & .69 & .48 \\
62. La preocupación de los miembros de su familia unos con otros & .64 & .41 \\
\hline Valores eigen & 4.67 & \\
\% de varianza & 46.7 & \\
\hline
\end{tabular}

Tabla 10

Matríz de intercorrelaciones y coeficiente alfa para las escalas FACES IV abreviado al Uruguay.

\begin{tabular}{|c|c|c|c|c|c|c|c|}
\hline Escalas & & 1 & 2 & 3 & 4 & 5 & 6 \\
\hline 1. & Cohesión balanceada & .71 & & & & & \\
\hline 2. & Enmarañada & .04 & .60 & & & & \\
\hline 3. & Desligada & $-.60^{* *}$ & .06 & .56 & & & \\
\hline 4. & Flexibilidad balanceada & $.40^{* *}$ & .02 & $-.27^{* *}$ & .46 & & \\
\hline 5. & Caótica & $-.37^{* *}$ & $.13^{*}$ & $.51^{* *}$ & $-.29^{* *}$ & .65 & \\
\hline 6. & Rígida & .05 & .08 & .09 & .07 & -.01 & .57 \\
\hline 7. & Satisfacción & $.62^{* *}$ & .11 & $-.60^{* *}$ & $.46^{* *}$ & $-.39^{* *}$ & .01 \\
\hline
\end{tabular}


etapas del análisis. En primer lugar, se analizó la validez de constructo del FACES IV usando la estructura de seis factores correspondiente al Modelo Circunflejo de los Sistemas Marital y Familiar, para replicar los resultados de Olson (2011). Los resultados obtenidos para la muestra uruguaya con el análisis factorial confirmatorio (utilizando los 42 ítems), muestran que el modelo no ajusta al original propuesto por Olson (2011), arribando a la misma conclusión obtenida en los procesos de adaptación de Italia, España y Hungría (Loriedo et al., 2013; Rivero et al., 2010; Mirnics et al., 2010).

Siguiendo la estrategia de análisis de datos propuesta por Rivero et al. (2010) en su proceso de adaptación, se continuó con el análisis de la estructura interna del cuestionario, agrupando los ítems según a qué escala pertenece cada uno en el modelo original. El trabajo con las dos escalas centrales balanceadas, evidenció resultados distintos para España y Uruguay. En el primero, Rivero et al. (2010) se encontró que los ítems de las escalas balanceadas centrales de cohesión y flexibilidad, respondían a una solución unidimensional, mientras que en el segundo, se encontró que los datos no ajustan a una solución undimensional. Eliminando tres ítems por escala, se logra un ajuste a un único factor y también a una estructura bifactorial. En consecuencia, se arribó a una conclusión distinta a la obtenida por Rivero et al. (2010). En España, se podrían usar ambas escalas juntas en un único factor que permite la evaluación global del funcionamiento familiar balanceado (cohesión y flexibilidad), cosa que no ocurrió en Uruguay, trabajando con todos los ítems.

Los resultados de los análisis con las escalas extremas o desbalanceadas, tanto en España como en Uruguay, reflejan la necesidad de trabajar por separado los extremos de las dimensiones flexibilidad (rígida / caótica) y cohesión (enmarañada / desligada). Estas escalas se adaptan bien al modelo propuesto por Olson (2011). Con la reespecificación del modelo tras la eliminación de ítems, alcanza un adecuado ajuste a un modelo de cuatro factores (desligada, enmarañada, rígida y caótica).

En suma, de los análisis anteriormente presentados, eliminando tres ítems en cada una de las dos escalas balanceadas y de las cuatro desbalanceadas, se arriba a seis escalas independientes, unidimensionales y con una fiabilidad aceptable. A continuación, con el modelo reespecificado, se logra mejorar el ajuste y confirmar una estructura de seis factores como la propuesta por los autores (Olson, 2011), arribando a una solución factorial semejante a la alcanzada por Rivero et al. (2010) en su versión abreviada a España. Ambas versiones cuentan con el mismo número de ítems, resultando en una versión final abreviada con 24 de los 42 ítems originales. En total, la versión uruguaya comparte con la versión española el $75 \%$ de los ítems de los 24 usados por Rivero et al. (2010).

Sin embargo, el índice de covarianza observado entre algunas de las dimensiones, tanto en los resultados españoles (Rivero et al., 2010), como en los nuestros, plantean dudas sobre la existencia de constructos claramente diferenciados. En España, se encontraron covarianzas muy altas entre las dimensiones cohesión y flexibilidad, no ocurriendo lo mismo en Uruguay, donde correlaciona en forma alta y positivamente (.67) pero más baja que lo reportado en España. Por otro lado, en Uruguay la correlación entre desligada y cohesión balanceada es muy alta, ocurriendo lo mismo que lo reportado por Olson (2011) y en la misma dirección que en el estudio español (Rivero et al., 2010). Por último, entre flexibilidad balanceada y caótica, tanto el estudio de Olson (2011) como el de Rivero et al. (2010), presentan correlaciones negativas y altas, pero en nuestro estudio la correlación es negativa y moderada. Específicamente, estas covarianzas altas encontradas entre las escalas cohesión balanceada y desligada, también encontradas por Olson (2011) y Rivero et al., (2010), plantean dudas sobre el constructo desligado como una dimensión discriminante. Esto pone en evidencia la necesidad de un estudio interno del instrumento para confirmar la estructura dimensional obtenida.

La fiabilidad de las escalas balanceadas de las dimensiones cohesión y flexibilidad presenta un buen índice en la escala cohesión $(\alpha=.71)$, con un resultado muy similar al español $(\alpha=.77)$. Sin embargo, en la escala flexibilidad balanceada se obtuvo una fiabilidad baja $(\alpha=.46)$ comparada con la reportada para España $(\alpha=.66)$. La fiabilidad de las escalas desbalanceadas (desligada / enmarañada; rígida / caótica) resulta adecuada, oscilando entre .57 y .65 , obteniendo así, resultados muy similares a los reportados en España que oscilan entre .51 a .74 (Rivero et al., 2010). En general, se han obteniendo adecuados índices de fiabilidad, con excepción de la escala cohesión balanceada tanto en España (.77) como en Uruguay (.71). El resto 
de los índices son moderadamente bajos en ambos países, si bien aceptables teniendo en cuenta la reducción significativa del número de ítems. La fiabilidad reportada por Olson (2011) se encuentra en un rango de .77 a .89 con escalas de siete ítems. En la adaptación española (Rivero et al., 2010) con una versión abreviada de cuatro ítems se obtuvieron fiabilidades en un rango de .51 a .77 . Nuestros resultados arrojaron fiabilidades similares pero levemente inferiores a las encontradas por los españoles, en un rango de .46 a .71. Es importante destacar que la longitud de la escala es uno de los factores que afectan la fiabilidad, al disminuir en tres los ítems por escala, es esperable que ésta disminuya. Entonces, al aumentar el número de ítems por escala, se aumentaría la fiabilidad (Barbero, Vila y Suárez, 2006). Por lo tanto, con solo cuatro ítems por escala se puede considerar que presenta una fiabilidad aceptable. De aumentar la cantidad de ítems a siete como la original, la fiabilidad calculada por el método de Spearman-Brown mejoraría, oscilando en un rango de .60 a .81 .

La correlación alta encontrada en el AFC, entre las escalas balanceadas de cohesión y flexibilidad $(r=.40)$ remite a la concordancia entre los dos conceptos, a mayor flexibilidad mayor cohesión familiar y a menor flexibilidad menor cohesión familiar (Olson y Gorall, 2003). En esta línea, las correlaciones entre la escala Satisfacción Familiar con las dos escalas centrales balanceadas, son positivas y fuertes, lo que confirma la concordancia del modelo: a mayor cohesión familiar (conexión) mayor flexibilidad (adaptabilidad) y mayor satisfacción familiar. Desde las hipótesis del modelo (Olson y Gorall, 2003), es esperable, dadas las características de las familias participantes (seleccionadas en instituciones educativas), obtener puntajes altos en las dos escalas centrales balanceadas (cohesión y flexibilidad) y puntajes bajos en las cuatros escalas desbalanceadas que evalúan los extremos de las dimensiones cohesión (enmarañada y desligada) y flexibilidad (rígida y caótica). Desde este punto de vista, notamos que las escalas desbalanceas enmarañada y rígida, correlacionan positivamente, la primera con cohesión balanceada $(r=.04)$ y la segunda con flexibilidad balanceada $(r=.07)$, contradiciendo así lo esperado por las hipótesis desarrolladas por Olson y Gorall (2003). Por el mismo procedimiento, resulta esperable que el extremo más desligado de las familias no fuera captado. Esto puede explicar la diferencia entre los resultados encontrados y lo que esperábamos encontrar según lo planteado por el Modelo Circunflejo.

En el estudio de validez concurrente, se encontró que la escala Satisfacción familiar correlaciona positivamente con las dos escalas centrales balanceadas (cohesión y flexibilidad) y negativamente con las escalas desbalanceadas desligada y caótica. Por otro lado, no correlaciona con las escalas enmarañada y rígida, siendo otro punto a continuar investigando. En este punto, encontramos coincidencias con el estudio español de Rivero et al. (2010).

La principal fortaleza de este estudio, consiste en que se trabajó a partir de una muestra de familias, por ser la población diana del instrumento. Este hecho ofrece garantía para que esta versión, pueda ser de gran utilidad para aproximarse a una evaluación más ajustada del funcionamiento familiar de la que ofrecian las anteriores versiones del FACES.

Consideramos que la versión uruguaya del FACES IV es un instrumento que puede resultar útil en la investigación sobre el funcionamiento familiar ya que la fiabilidad adecuada a estos efectos es la que está en el entorno a .70. Cuando el objetivo es diagnóstico o clasificación, la fiabilidad mínima debe ser de .80 (Montero y León, 2002). Por este motivo, para su uso con el objetivo diagnóstico y aplicación en la clínica, habrá que continuar investigando sobre la validez de contenido.

Recordamos que sólo trabajamos con el cuestionario de autoadministrado que evalúa el Modelo Circunflejo. Nuestra información sería más precisa si la complementáramos con la perspectiva externa del funcionamiento familiar evaluada por un terapeuta, proporcionada por la Clinical Rating Scale. Esto nos permitiría unir la investigación con la práctica clínica, permitiéndonos desarrollar nuevos ítems adaptados a nuestra cultura, mejorando la fiabilidad del instrumento.

En suma, se sugiere no dar por terminado el proceso de adaptación del FACES IV al Uruguay hasta no volver al estudio de validez de contenido para las escalas desbalanceadas enmarañada y rígida. Se necesitaría buscar nuevas formas de operacionalizar en ítems los conceptos semánticos de estas dos escalas y traducirlos en nuevos ítems, procurando se adapten con mayor precisión a la cultura de Uruguay y de la región, para volver a comenzar los estudios de dimensionalidad y validez. 
Se sugiere también que, una vez terminado el proceso de adaptación del FACES IV al Uruguay y mejoradas sus propiedades psicométricas, se aplique a diferentes poblaciones clínicas, tales como familias que viven problemáticas de consumo de drogas, abuso infantil, trastornos alimentarios, entre otros, como medio de realizar diagnósticos precisos y evaluaciones fundamentadas de las intervenciones clínicas.

\section{Referencias}

Baiocco, R., Cacioppo, M., Laghi, F., \& Tafa, M. (2012). Factorial and Construct Validity of FACES IV Among Italian Adolescents. Journal of Child and Family Studies , 1-9.

Barbero, M. I., Vila, E. y Suárez, J. C. (2006). Psicometría. Madrid: UNED.

Bentler, P. M., \& Bonnet, D. C. (1980). Significance Tests and Goodness of Fit in the Analysis of Covariance Structures. Psychological Bulletin , 88 (3), 588-606.

Brown, P. (2011). Alexitimia y trastornos de la alimentación: estudio empírico con una muestra de población clínica y sus padres en la ciudad de Montevideo (Memoria de grado). Universidad Católica del Uruguay.

Carretero-Dios, H., \& Pérez, C. (2007). Standards for the development and review of instrumental studies: Considerations. International Journal of Clinical and Health Psychology , 7 (3), 863-882.

Cattell, R. B. (1966). The scree test for the mumber of factors Multivariate Behavioral Reserch. (1), 245-276.

Costa Ball, D., González Tornaría, M., Masjuan, N., Trápaga, M., del Arca, D., y Scafarelli, L. (2009). Escala de evaluación del funcionamiento familiar -FACES IV-: Proceso de adaptación a Montevideo, Uruguay. Ciencias Psicológicas , 3 (1), 43-56.

Costa Ball, D., Masjuan, N., González Tornaria M.,Trápaga, A., Scafarelli, L., del Arca, D. y Feibuscheurez, A. (Marzo, 2010). Adaptation for Uruguay of Family Adaptability and Cohesion Evaluation Scales (FACES IV): A Prelimanary Statistical Study. Simposio llevado a cabo en XVIII World International Family Therapy Association (IFTA) Congress, Buenos Aires.

del Arca, D. (2010). Funcionamiento Familiar y rendimiento académico en familias con hijos cursando secundaria en un colegio católico de Montevideo (Memoria de grado). Universidad Católica del Uruguay.

Feibuscheurez, A. (2009). Características del funcionamiento familiar en el que un miembro padece un trastorno de la conducta alimentaria. Aproximaciòn desde el Modelo Circunflejo (Memoria de grado). Universidad Católica del Uruguay.

Horn, J. L. (1965). A rationale and test for sthe mumber of factors in factor analysis. Psychometrika , 179-185.

Hu, L.-t., \& Bentler, P. M. (1999). Cutoff Criteria for Fit Indexes in Covariance Structure Analysis: Conventional Criteria Versus New Alternatives. Structural Equation Modeling, $6(1), 1-55$.

Kouneski, E. (2000). The Family Circumplex Model, FACES II, and FACES III: Overview of Research and Aplications. Recuperado de http://www.facesiv.com/pdf/ faces_and_circumplex.pdf
Koutra, K., Trilivia, S., Rouneliataki, T., Lionis, C., \& Vgontzas, A. (2012). Cross-Cultural Adaptation and Validation of the Greek Version of the Family Adaptability and Cohesion Evaluation Scales IV Package (FACES IV Package). Journal of family Issues . doi:10.1177/0192513X12462818

Lestido, F. (2013). Funcionamiento familiar en una muestra de familias con un hijo que presenta Trastorno del Espectro Autista (Memoria de grado). Universidad Católica del Uruguay.

Lévy, J. P., Martín, M. T., y Román, M. V. (2006). Optimización según estructuras de covarianzas. En J. P. Lévy y J. Varela (Eds.), Modelización con Estructuras de Covarianzas en Ciencias Sociales (pp. 11-30). Madrid: Netbiblo.

Loriedo, C., Di Nuovo, S., \& Visani, E. (2013). FACES-IV: Italian reliability and validity. Recuperado de http://www. buildingrelationships.com/pdf/italy_facesiv_2013.pdf

MacCallum, R. C., Browne, M. W., \& Sugawara, H. M. (1996). Power Analysis and Determination of Sample Size for Covariance Structure Modeling. Psychological Methods, 1(2), 130-149.

Maglio, A. L., y Schmidt, V. (2010). Los instrumentos de evaluación del funcionamiento familar. En N. Leivobich, V. Schmidt y Otros, Ecoevaluación Psicológica del Contexto Familiar (pp. 103-108). Buenos Aires: Guadalupe.

Massonnier, N. (2011). Comunicación y satisfacción en las familias de estudiantes de la UCU, según estos sean migrantes del interior o vivan con sus padres en Montevideo (Memoria de Grado) Universidad Católica del Uruguay.

McGoldrick, M., Carter, B., \& Garcia Preto, N. (2011). Overview: The Life Cycle in its Changing Context. En B. McGoldrick, B. Carter \& N. Garcia Preto (Eds.), The Expanded Family Life Cycle: Individual, Family, and Social Perspectives (4a ed., pp. 1-19). Boston: Allyn \& Bacon.

Mirnics, Z., Vargha, A., Tóth, M., \& Bagdy, E. (2010). CrossCultural Applicability of FACES IV. Journal of Family Psychotherapy, 21, 17-33.

Montero, I., y León, O. G. (2002). Clasificación y descripción de las metodologías de. Revista Internacional de Psicología Clínica y de la Salud , 2 (3), 503-508.

Muñiz, J., Elosua, P., y Hambleton, R. K. (2013). Directrices para la traducción y adaptación de los tests: segunda edición. Psicothema, 25 (2), 151-157.

Oliva, E., yArranz, E. (2011). Nuevas familias y bienestarinfantil. Sevilla: Publicaciones de la Unversidad de Sevilla.

Olson, D. H. (1995). Family Satisfaction Scale. MN: Life Innovations.

Olson, D. H. (2000). Circumplex model of family systems. Journal of Family Therapy, 22 (2), 144-167.

Olson, D H. (2011). FACES IV and the Circumplex Model: Validation Study. Journal of Marital \& Family Therapy 3 (1), 64-80.

Olson, D. H.; Candyce, S. R., \& Sprenkle, D. H. (1989). Circumplex Model: Systemic Assessment and Treatment of Families. New York: The Harworth Press.

Olson, D. H., \& Gorall, D. M. (2003). Circumplex Model of Marital and Family Systems. En F. Walsh, Normal Family Processes (3a ed., pp. 514-547). New York: The Guilford Press.

Olson, D. H., \& Gorall, D. M. (2006). FACES IV \& the Circumplex Model. Minnesota: Life Innovations. 
Olson, D. H., Portner, J., \& Bell, R. Q. (1982). FACES II. St. Paul: University of Minnesota.

Olson, D. H., Portner, J., \& Lavee, Y. (1985). FACES III. St. Paul: University of Minnesota.

Olson, D. H., Sprenkle, D. H., \& Russell, C. S. (1979). Circumplex model of marital and family systems, I: Cohesion and adaptability dimensions, family types and clinical applications. Family Process , 18, 3-28.

Rivero, N., Martínez-Pampliega, A., \& Olson, D. H. (2010). Spanish Adaptation of the FACES IV Questionnaire: Psychometric Characteristics. The Family Journal: Counseling and Therapy for Couples and Families, 18 (3), 288-296.

Rodrigo, M. J., Máiquez, M. L., Martín, J. C., y Byrne, S. (2008). Preservación Familiar. Un enfoque positivo para la interpretación con familias. Madrid: Pirámide.

Sanz, M., Iraurgi, I., y Martínez-Pampliega, A. (2002). Evaluación del funcionamiento familiar en toxicomanías: adaptación española y características de adecuación métrica del FAP-FACES IV. En I. Iraurgi, \& F. Zonzález, Instrumentos de evaluación en drogodependencias (pp. 404-434). Madrid: Aula Médica.
Schmidt, V., y Barreyro, J. P. (2010). Proceso de Adaptación de la escala a nuestra población Propiedades Psicométricas de la versión argentina. En N. B. Leibovich, V. Schmidt y Otros, Ecoevaluación Psicológica del Contexto Familiar (pp. 117-130). Buenos Aires: Guadalupe.

Steiger, J. H. (2007). Understanding the limitations of global fit assessment in structural equation modeling. Personality and Individual Differences , 42 (5), 893-898.

Urrutia, A. M. (2012). Funcionamiento familiar donde hay un miembro con TDAH: aproximación desde el modelo circunflejo (Memoria de grado). Universidad Católica del Uruguay.

Wagner, A. (2011). Desafios Psicossociais da Família Contemporânea: Pesquisas e Reflexões. Porto Alegre: Grupo A.

Walsh, F. (2004). Resiliencia familiar. Estrategias para su fortalecimiento. Buenos Aires: Amorrortu.

\section{Apéndice}

\section{FACES IV - Versión uruguaya}

Instrucciones para los miembros de la familia:

Todos los miembros de la familia mayores de 12 años deben completar el FACES IV independientemente, sin consultar o discutir sus respuestas.

Rodee con un círculo el número correspondiente en el espacio brindado en el protocolo.

\footnotetext{
1. Totalmente en desacuerdo

2. Generalmente en desacuerdo

3. Indeciso

4. Generalmente de acuerdo

5. Totalmente de acuerdo
}

1. Los miembros de nuestra familia están involucrados, se interesan y participan en la vida de sus integrantes. (CB)

2. Nuestra familia intenta nuevas formas de lidiar con los problemas. (FB) 3. Los miembros de nuestra familia se sienten más cercanos a personas externas a la familia que a los propios miembros de la familia. (D)

4. Pasamos demasiado tiempo juntos. (E)

5. Hay consecuencias estrictas por romper las reglas en nuestra familia. (R)

6. En nuestra familia, parecería que nunca nos organizáramos. (C)

7. Los miembros de nuestra familia se sienten muy cercanos unos a otros. (CB)7

11. Hay consecuencias claras cuando un miembro de la familia hace algo mal. (R)
12. Es difícil saber quien manda en nuestra familia. (C) 13. Los miembros de la familia se apoyan mutuamente unos a otros durante los periodos difíciles. (CB)

14. La disciplina es justa en nuestra familia. (FB)

16. Los miembros de la familia dependen demasiado unos de otros. (E) 17. Nuestra familia tiene una regla para casi toda situación posible. $(R)$ 18. En nuestra familia las cosas que nos proponemos no se hacen. (C) 20. Mi familia es capaz de ajustarse a los cambios cuando es necesario. (FB)

22. Los miembros de la familia tienen poca necesidad de tener amigos fuera de la familia. (E)

26. Nos turnamos las tareas del hogar entre nosotros. (FB)

27. Rara vez nuestra familia hace cosas junta. (D)

28. Nos sentimos demasiado juntos unos a otros. (E)

31. Aunque los miembros de la familia tienen intereses individuales, aún participan de las actividades familiares. (CB)

33. Los miembros de la familia raras veces se apoyan entre sí. (D) 41 Una vez que una decisión está tomada, es muy difícil modificar esa decisión. $(R)$

42. Nuestra familia se siente agitada y desorganizada. (C)

Nota: $C B=$ Cohesión Balanceada; $F B=$ Flexibilidad Balanceada; $D=$ Desligada; $E=$ Enmarañada; $R=$ Rígida $; C=$ Caótica .

Para citar este artículo:

Costa Ball, D., González Tornaría, M., del Arca, D., Masjuan, N. y Olson, D.H. (2013). Propiedades Psicométricas del FACES IV: Estudio de validez en población uruguaya. Ciencias Psicológicas VII (2): $119-132$. 